Check for updates

Cite this: Mater. Chem. Front., 2019, 3, 1113

Received 8th March 2019, Accepted 28th April 2019

DOI: 10.1039/c9qm00144a

rsc.li/frontiers-materials

\section{Folic acid functionalized hollow nanoparticles for selective photodynamic therapy of cutaneous squamous cell carcinoma $\dagger$}

\author{
Duncan Hieu M. Dam, (D) a Lingzhi Zhao, ${ }^{\text {bc }}$ Sophia A. Jelsma, (D) ${ }^{a}$ Yanli Zhao (D)*b \\ and Amy S. Paller (D) *a
}

Intervention for non-melanoma skin cancer (NMSC) remains largely surgical, and development of an effective, tissue-salvaging, less invasive therapy is a significant unmet need. Photodynamic therapy is a non-invasive approach for NMSC, but depends on the uptake of protoporphyrin IX (PphIX), a naturallyoccurring photosensitizer. However, the bioavailability of PphIX is low and improved delivery is needed. Nanoscale carriers can facilitate therapeutic delivery, including through the epidermal barrier. We have developed ultra-small hollow silica nanocarriers (HSdots) $(\sim 10 \mathrm{~nm})$ packed with zinc phthalocyanine ( $\mathrm{nnPC}$ ), a porphyrin that can be excited by near infrared light (671 nm laser). The nanocarrier has demonstrated the ability to deliver to the epidermis of human intact skin. To provide selective capability of ZnPC-loaded HSdots to cutaneous squamous cell carcinoma (SCC) regions, we conjugated folic acid on the surface of HSdots to target the folic acid (FA) receptor, which we found to be more highly expressed in human cutaneous and head/neck SCC lines (A431, SCC12, CAL27) and SCC tissues than in normal human epidermal keratinocytes (NHEKs) and normal skin, respectively. As shown by inductively coupled plasma-optical emission spectrometry, uptake of ZnPC-loaded FA-HSdots in SCC cells after 48 hours was higher than in NHEKs (4- to 5-fold more Si and 6- to 7-fold more Zn). Treatment with the 1-4 $\mathrm{mg} \mathrm{mL}^{-1} \mathrm{ZnPC}$-loaded FA-HSdots (0.01-0.04 $\left.\mathrm{mg} \mathrm{mL}^{-1} \mathrm{ZnPC}\right)$ led to concentration-dependent toxicity of SCCs after exposure to $671 \mathrm{~nm}$ laser for $2 \mathrm{~min}$. ZnPC-loaded FA-HSdots (4 mg mL $\mathrm{m}^{-1}$ ) led to $\geq 90 \%$ SCC death after one laser exposure, accompanied by a 2-3-fold increase in caspase 3 expression and increased nuclear double-stranded DNA breaks, suggesting apoptosis. No toxicity was observed in NHEKs or in SCCs treated with only laser or only the ZnPC-loaded FA-HSdots. Treatment with laser and $\mathrm{ZnPC}-\mathrm{HS}$ dots without FA receptor targeting led to toxicity in NHEKs that was indistinguishable from that in SCCs, suggesting the importance of FA to selectively target SCCs. Nanocarriers are a promising tool to traverse the epidermal barrier, allowing topical delivery of ZnPC-loaded FA-HSdots and ZnPC/chemotherapy for skin cancer.

\section{Introduction}

\footnotetext{
${ }^{a}$ Department of Dermatology, Feinberg School of Medicine, Northwestern University, Chicago, IL, 60611, USA. E-mail: apaller@northwestern.edu

${ }^{b}$ Division of Chemistry and Biological Chemistry, School of Physical and Mathematical Sciences, Nanyang Technological University, 637371, Singapore. E-mail: zhaoyanli@ntu.edu.sg

${ }^{c}$ State Key Laboratory of Natural Medicine, School of Basic Medical Sciences and Clinical Pharmacy, China Pharmaceutical University, Nanjing, Jiangsu 211198,

P. R. China

$\dagger$ Electronic supplementary information (ESI) available: Penetration of nanocarrier HSdots through human intact skin, uptake of ZnPC-loaded HSdots in SCC and normal cells, toxicity of ZnPC-loaded HSdots after laser exposure, expression of folate receptors in different SCC tissues, laser setup for laser exposure, effect of ZnPC-loaded FA-HSdots 24 hours after laser exposure, TUNEL assay in CAL27 cells, and compared therapeutic efficacy of ZnPC-loaded HSdots with commercially available products. See DOI: 10.1039/c9qm00144a
}

Non-melanoma skin cancer (NMSC), particularly basal cell and squamous cell carcinomas (BCCs and SCCs), are the most common malignant tumors of humans. The incidence rate of NMSC is increasing, ${ }^{1-3}$ including among organ transplant recipients (who are at a 65-fold risk of developing skin squamous cell carcinomas)., NMSC is largely treated with surgical excision, leaving often unsightly and sometimes uncomfortable scars. In recent decades, photodynamic therapy (PDT) has emerged as an effective technique for the treatment of selected skin diseases, and has shown early evidence of being a new non-invasive strategy for treating NMSC. ${ }^{6,7}$ Three indispensable components are required for PDT: the photosensitizer (PS), which must penetrate into skin, excitation light, and oxygen. During the process of PDT, the PS is 
located inside the targeted cancer cells or tumor tissues. The excitation light with an appropriate wavelength is administrated to activate the PS, and then the energy is transferred from the excited PS in a triplet state $\left({ }^{3} \mathrm{O}_{2}\right)$ to the neighboring oxygen molecules to convert them into reactive oxygen species (ROS), such as singlet oxygen $\left({ }^{1} \mathrm{O}_{2}\right)$. The generated ROS can lead to the apoptosis or necrosis of cancer cells, thus promoting the destruction of tumor tissues. ${ }^{8-10}$

While PDT is promising, delivery of the PS remains an issue. The majority of PS compounds are hydrophobic with limited aqueous solubility and low tissue-penetration capability. Upon administration, these hydrophobic PS molecules tend to aggregate, resulting in poor generation of ${ }^{1} \mathrm{O}_{2}$ and inadequate pharmacokinetics. In addition, the generated ROS can damage normal cells, suggesting the need to selectively target abnormal cells. Although effort has been expended to modify PS with covalently linked receptors that are over-expressed in cancer cells, PS accumulation in subcellular localizations is still insufficient. ${ }^{6}$ To improve the therapeutic efficiency of PDT and avoid its potential side effects, targeted administration and concentrated delivery of PS using carriers are necessary. Current PDT treatment uses 5-aminolaevulinic acid (5-ALA) or methyl aminolaevulinate (MAL), which requires UV-light to generate porphyrin IX (PphIX). ${ }^{11}$ PphIX is considered to be a promising PS for PDT, including in NMSC. ${ }^{5}$ PphIX is the immediate precursor of heme in the biosynthetic pathway, which can be produced by 5-ALA in mitochondria. ${ }^{7,12}$ The metabolic process includes the formation of porphobilinogen (PBG) from 5-ALA, followed by the production of PphIX catalyzed by PBG-deaminase, and then PphIX is converted to heme through ferrochelatase, thus losing its function as PS. In rapidly proliferating cancer cells, enhancing PBGdeaminase and inhibiting ferrochelatase activity can theoretically increase the PphIX concentration, ${ }^{9}$ contributing to selective accumulation and concentrated delivery of PphIX in NMSC tumor tissue for PDT. Although effective, 5ALA-PDT or MALPDT use is limited, since longer wavelength UVA and blue visible light (400-490 $\mathrm{nm}$ ) can only penetrate up to $1 \mathrm{~mm}$ and thus cannot reach deeper skin areas. ${ }^{13}$ Thus, direct delivery of PphIX instead of 5-ALA or MAL can significantly improve accumulation of PphIX in cancer cells. Furthermore, PphIX for PDT is activated by a more advantageous wavelength (i.e., $>600 \mathrm{~nm}$ to enable $4-6 \mathrm{~mm}$ depth penetration), which can reach the dermis. ${ }^{14}$

However, poor aqueous solubility and low tissue-penetration capability of PphIX limit its application in clinical PDT. Surface bacteria also have the capacity to engulf PphIX, as has been shown to occur in the GI track, resulting in inaccurate endoscopic observations. ${ }^{6}$ Hence, a delivery vehicle is needed to facilitate PphIX bypass of the lipophilic barrier for cell entry, while protecting PphIX from being engulfed by bacteria. ${ }^{7,9,15}$ In addition, the ideal agent for treating NMSC should accumulate in tumor long enough for PDT treatment, but then be cleared. Nanoscale materials have increased the efficacy of previously established diagnostic and therapeutic regimens because of improved delivery. ${ }^{16-20}$ While PphIX itself is non-toxic and is metabolized, the nanocarriers are foreign materials and, in animal studies, some have been shown to be retained for long periods in viscera, particularly the liver and spleen, ${ }^{21}$ leading to potential organ damage. Thus, we studied the use of a nanoparticlebased delivery system that encapsulates PphIX into nanoparticles for more targeted delivery to tumor cells, but are rapidly excreted.

We have recently developed ultra-small silica nanoparticles capable of serving as nanocarriers. ${ }^{22-25}$ Silica materials are commonly considered biodegradable materials in the biological environment. ${ }^{26,27}$ In this study, we introduced a hollow ultrasmall silica nanoparticle as a nanocarrier for targeted topical delivery of photosensitizer zinc phthalocyanine (ZnPC) to cutaneous squamous cell carcinomas (SCCs) for photodynamic skin cancer therapy. The silica nanoparticles were synthesized in acidic conditions and retained a considerable amount of silanol groups, which facilitates their degradation. ${ }^{28}$ Because early studies showed that ZnPC-loaded HSdots are taken up well by both SCC cells and normal human epidermal keratinocytes (NHEKs), we modified the ZnPC-loaded HSdots with folic acid (ZnPC-loaded FA-HSdots) for selective uptake of the nanoconstructs in SCC cells and reduced toxicity to NHEKs. PDT treatment of SCC cells with $671 \mathrm{~nm}$ near infrared laser and ZnPC-loaded FA-HSdots resulted in $>90 \%$ reduction of the cancer cells with no adverse effect on NHEKs. Ultimately, this study provides an innovative therapeutic platform using PDT which has the potential to be applied topically and selectively lead to tumor cell toxicity.

\section{Results and discussion}

\section{Non-selective treatment of ZnPC-loaded nanodots in squamous cell carcinoma}

To evaluate whether HSdots can function as a nanocarrier for topical application, we topically applied a mixture of $12.5 \mathrm{mg} \mathrm{mL}$ of TRITC-tagged HSdots with the commonly used moisturizer, Aquaphor $\mathbb{C},(1: 1)$ onto the surface of human intact abdominoplasty skin. TRITC signal was observed in the epidermis after 72 hours of incubation, suggesting that the HSdots can penetrate to the epidermal layer of human intact skin (Fig. S1, ESI $\dagger$ ). This result affirms that HSdots are suitable nanocarriers for topical application. We then synthesized ZnPC-loaded nanodots (ZnPCloaded HSdots) and tested their delivery into SCC cells; ZnPC was chosen as the photosensitizer due to its excitation (at $671 \mathrm{~nm}$; close to the near infrared region), which provides deeper laser exposure and a greater likelihood of reaching more invasive forms of cutaneous SCC. We observed abundant uptake of ZnPC-loaded TRITC-labeled HSdots (2 $\mathrm{mg} \mathrm{mL}^{-1}$ ) after a 48 hour incubation with SCC cells (Fig. S2A and B, ESI $\dagger$ ). However, we also observed an equivalent uptake of ZnPCloaded HSdots in our NHEKs (Fig. S2C, ESI $\dagger$ ). Furthermore, cell exposure to laser after treatment with ZnPC-loaded HSdots $\left(2 \mathrm{mg} \mathrm{mL}^{-1}\right)$ resulted in $60 \%$ cell death within $2 \mathrm{~h}$ and $80-90 \%$ cell death within $24 \mathrm{~h}$ after exposure of SCCs, but also $>80 \%$ cell death after $24 \mathrm{~h}$ in ZnPC-loaded NHEKs (Fig. S3, ESI $\dagger$ ). These results emphasized the need for greater selectivity in uptake by SCCs than NHEKs to minimize normal cell toxicity. 


\section{SCC cell lines/SCC tissues express more folate receptor than normal keratinocytes/skin}

To identify a new strategy for generating ZnPC-loaded HSdots that selectively target SCCs, we explored the differential expression of the folate receptor (FR), which has previously been noted to be highly overexpressed in many tumor types. ${ }^{23}$ Western blot analysis showed an approximately 2-fold increase in FR expression in epidermal (A431, SCC12) and head and neck (CAL27) SCC lines, compared to NHEKs (Fig. 1A). As shown in Fig. 1B, FR expression was undetectable or barely detected in the epidermis (area within the dotted lines) of male or female normal human abdominoplasty skin by confocal immunofluorescence after treatment with anti-FR antibodies. Furthermore, immunofluorescence staining of keratin 16 (K16), which marks hyperproliferative epidermis, also showed minimal signal in normal epidermis. In contrast, both FR and K16
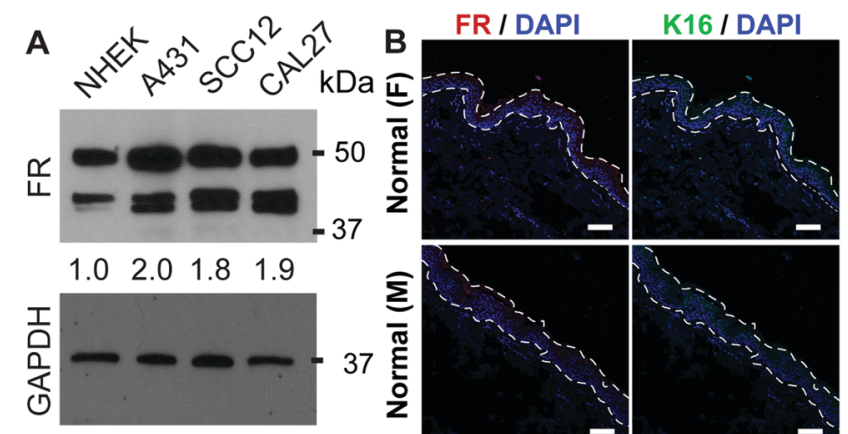

C FR I DAPI
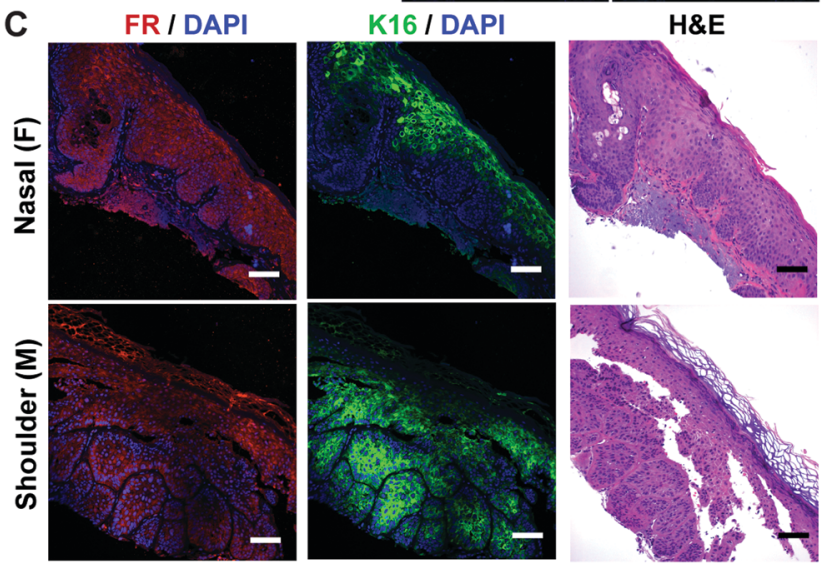

Fig. 1 Expression of the folate receptor on SCC cells and tissues. (A) Approximately 2-fold increase in total expression of folate receptors (FR) (top band: alpha-chained FR, bottom band: beta-chained FR) in SCC cells compared to NHEKs, as detected by western blotting. Band densitometry was calculated by normalizing the total densitometry of both FR bands to GAPDH. (B) 6 normal skin tissues ( 3 male and 3 female) were used to assess FR expression in normal skin. Representative images of immunofluorescence detection, showing minimal to no staining for FR (red) or keratin 16 (K16, green), a marker for hyperproliferation, in normal human skin. The blue stain, DAPI, shows the presence of the epidermal cells. (C) FR expression in 12 SCC tissues from various body sites were evaluated. Immunofluorescence detection of FR and K16 using confocal microscopy, showing the correlation of increased FR and K16 in the SCC tissue. Hematoxylin and eosin (HEE) stained section of human cutaneous SCCS is shown at the right. There was no difference in female vs. male cancers. Scale bar: $50 \mu \mathrm{m}$. were strongly expressed in the SCCs from various head and neck sites including SCC tissues from ears (female), scalp (female), nasal (male) and shoulders (male) (Fig. 1C and Fig. S4, ESI $\dagger$ ), regardless of patient sex, including in the dysplastic, hyperproliferative epidermis associated with the SCC tissues. We also noticed increased FR as well as K16 expression in the perilesional epidermis adjacent to SCC, suggesting that these lesions are also not completely normal. The increased FR expression allows for a strategy to deliver drugs selectively to SCCs and dysplastic epidermal tissues.

\section{Synthesis of folate receptor targeted, hollowed silica nanodots loaded with photosensitizer}

To create nanodots that selectively target the folate receptor on SCC cells, targeting ligand folic acid (FA) was chosen for conjugation with F108 through the esterification reaction between the carboxylic acid group of FA and the terminal hydroxyl group of F108 (PEO132-PPO50-PEO132). A doping ratio of 5\% for FA-F108 to F108 was adopted to synthesize FA-HSdots with FA as targeting ligands. Taking the modification ratio of FA to F108 into account $(85 \%)$, the amount of FA on the terminal of the ligands can be estimated as $4.3 \%$. We then fabricated hollowed silica-polymer hybrid nanodots (HSdots) using triblock copolymer F108 and silica precursor (Fig. 2A and B). The polymer blocks formed a micelle, which was swelled by the pore-expanding agent, cyclohexane, and then cross-linked by the silica shell. Thus, the hydrophobic PPO domains are located inside the silica shell, and the hydrophilic PEO domains branch from the silica shell in a dense concentration (0.54 PEG per $\mathrm{nm}^{2}$ ) and endow the nanoparticle with "stealth" properties. The brush-like PEG conformation promotes resistance to opsonization and uptake of macrophages. ${ }^{29}$

The poly( $p$-phenylene oxide) (PPO) blocks form an inner polymeric core, which can be used to load hydrophobic photosensitizers such as ZnPC. The solid silica shell outside the PPO core can tightly hold the surfactant molecules to maintain the micelle structure. Cyclohexane was employed as a pore-expanding agent to create the hollow structure in the hybrid nanoparticles, and can be completely removed from the resulting product by rotary evaporation. The capability of HSdots to load hydrophobic guest molecules were tested using pyrene as a model molecule. Our previous results showed that the ratio between the first fluorescence peak at $374 \mathrm{~nm}$ and the third peak at $384 \mathrm{~nm}$ of pyrene changed significantly after the addition of HSdots to the solution, indicating that the pyrene molecule was located in a very hydrophobic environment. ${ }^{28}$ This result directly demonstrated the pyrene can penetrate the silica shell and get into the hydrophobic PPO core of HSdots, which implies that the polymer does not block the hollow pore. In our previous paper, we characterized the pore size and pore volume of HSdots after the removal of the polymer F108, and found a large pore volume of $0.78 \mathrm{~cm}^{3} \mathrm{~g}^{-1}$ and a narrow pore size distribution peaked at $7.1 \mathrm{~nm}^{28}$

Transmission electron microscopy (TEM) images of ZnPCloaded HSdots and ZnPC-loaded FA-HSdots showed that both nanoparticles are monodispersed hollow spheres, with an average diameter of $28 \mathrm{~nm}$ and an inner pore size of $10 \mathrm{~nm}$ 
A

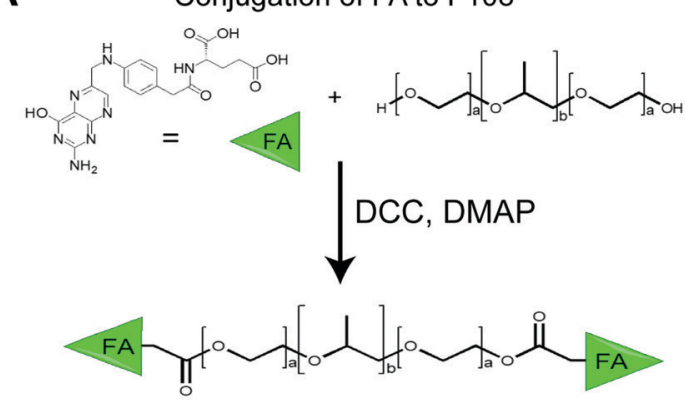

B

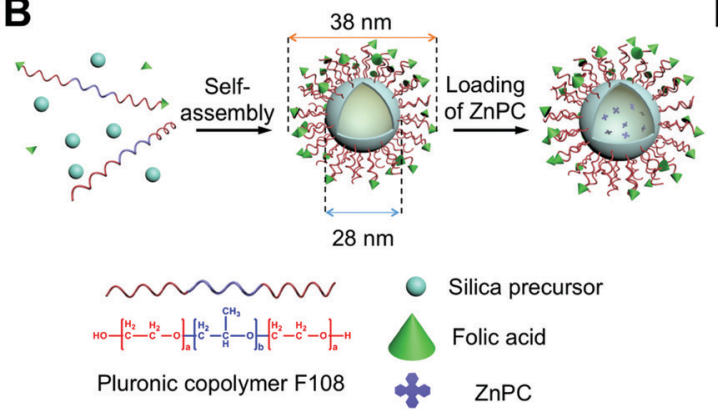

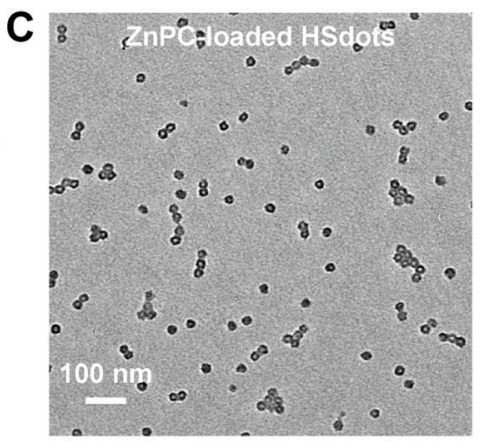

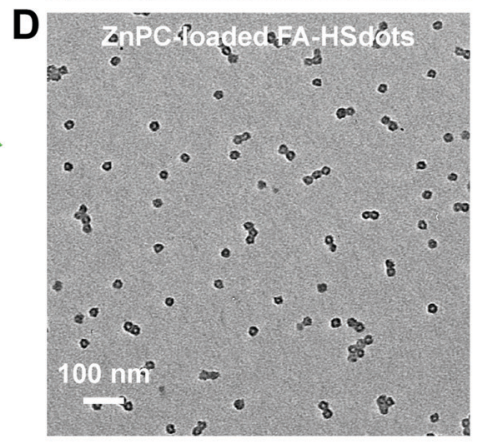

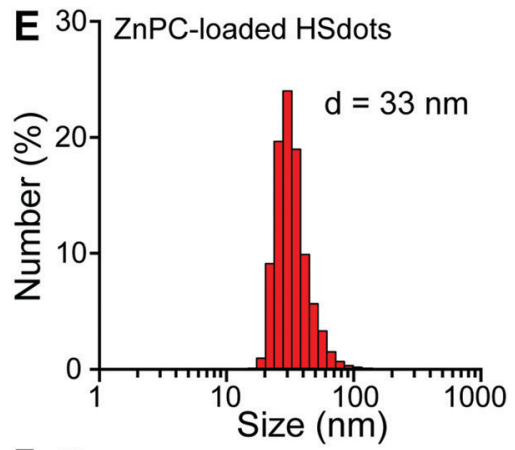

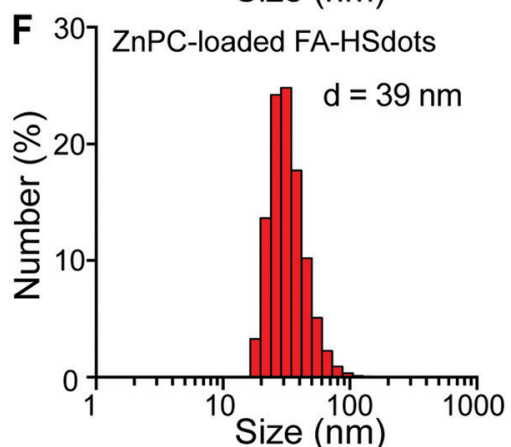

Fig. 2 Synthesis of folic acid conjugated hollow silica dots loaded with ZnPC. (A) Folic acid (FA) was conjugated with F108 through the esterification reaction between the carboxylic acid group of FA and the terminal hydroxyl group of F108. (B) Hollowed nanoparticles were produced through the selfassembly between silica precursor and F108 doped with FA-modified F108, and ZnPC was loaded on the particles. (C) TEM image of ZnPC loaded HSdots. (D) TEM image of ZnPC-loaded FA-HSdots. (E and F) Hydrated size of the nanoparticles was determined using dynamic light scattering.

(Fig. 2C and D). When hydrated, the ZnPC-loaded HSdots measured approximately $36 \mathrm{~nm}$ in diameter and the ZnPCloaded FA-HSdots $38 \mathrm{~nm}$, based on dynamic light scattering measurement (Fig. 2E and F). The discrepancy between the particle size and hydrated size can be attributed to the PEG ligands outside of the silica shell.

\section{ZnPC-loaded FA-HSdots show selective uptake in SCC cell lines}

The cellular uptake efficiency of FA-containing nanoparticles was studied in both NHEKs and SCC lines in vitro. Uptake of TRITC-labeled ZnPC-loaded FA-HSdots at $1 \mathrm{mg} \mathrm{mL}{ }^{-1}$ HSdots (0.01 $\mathrm{mg} \mathrm{mL}^{-1} \mathrm{ZnPC}$ ) after 48 hours was easily observed in SCC12, A431 and CAL27 cells (Fig. 2B-D), but the signal was significantly lower in NHEKs (Fig. 3A). These observations indicated that the FA-containing nanoparticles could be selectively endocytosed by cancer cells.

We then used inductively coupled plasma-optical emission spectrometry (ICP-OES) to quantify the amount of silica and zinc taken up in these cells. All of the three tested concentrations (0.01-0.04 $\mathrm{mg} \mathrm{mL}^{-1} \mathrm{ZnPC}$ ) led to significantly higher $\mathrm{Zn}$ (4-5-fold more) and Si (6-7-fold more) update in all of the SCC lines vs. the NHEKs $(p<0.001)$ (Fig. 3E and F). These data further confirmed that ZnPC-loaded FA-HSdots were delivered more efficiently to SCC cells compared to normal keratinocytes, suggesting the ability to selectively deliver ZnPC using FA-conjugated nanocarriers to SCCs. In addition, ZnPC-loaded FA-HSdots are always stored in phosphate buffer saline (PBS) and no visible crashing of the nanomaterial was observed. Once treated in cell media containing $10 \%$ fetal bovine serum (FBS), we did not see any crashing of the nanomaterial within 48 hour treatment, indicating its good stability.

\section{Neither the ZnPC-loaded FA-HSdots nanocarrier nor the laser alone is toxic to SCC cells or NHEKs}

Toxicity was assessed through in vitro cell viability based on the 3-(4,5-dimethylthiazol-2-yl)-2,5-diphenyltetrazolium bromide (WST-1) assay. To activate the photosensitizing property of ZnPC and evaluate the effect of laser exposure, we selected a continuous-wavelength laser with a $671 \mathrm{~nm}$ wavelength as excitation source, which matched the absorption peak of ZnPC (Fig. S5, ESI $\dagger$ ). SCC12, A431, and CAL27 cell lines and NHEKs were seeded into 96-well plates and exposed to the $671 \mathrm{~nm}$ laser. Laser alone did not affect cell viability at $2.7 \mathrm{~mW} \mathrm{~cm}^{-2}$ or below (Fig. $4 \mathrm{~A}$ ) or for up to $4 \mathrm{~min}$ at $2.7 \mathrm{~mW} \mathrm{~cm}^{-2}$ (Fig. 4B), noting that this laser density is much lower that the currently-approved laser density for PDT treatment $\left(\sim 100 \mathrm{~mW} \mathrm{~cm}^{-2}\right) .{ }^{30}$ In addition, no toxicity was observed in SCC cells or NHEKs treated for $48 \mathrm{~h}$ with 2 to $4 \mathrm{mg} \mathrm{mL}^{-1}$ of the ZnPC-loaded FA-HSdots nanocarriers alone (Fig. 4C).

\section{In vitro PDT evaluation of ZnPC-loaded FA-HSdots}

To assess the efficiency of photosensitization by ZnPC-loaded FA-HSdots in cancer cells, 1 to $4 \mathrm{mg} \mathrm{mL} \mathrm{mL}^{-1}$ ZnPC-loaded FA-HSdots were added to the growth medium of SCC cells and NHEKs for $48 \mathrm{~h}$. After washing and substitution with phenolphthalein-free growth media (DMEM/F12 with 10\% FBS) to prevent laser excitation of phenolphthalein, cells were immediately exposed to $671 \mathrm{~nm}$ laser at $1 \mathrm{~mW}$ for $2 \mathrm{~min}$. Two hours after laser exposure, dose-dependent cell death was achieved in all SCCs, 

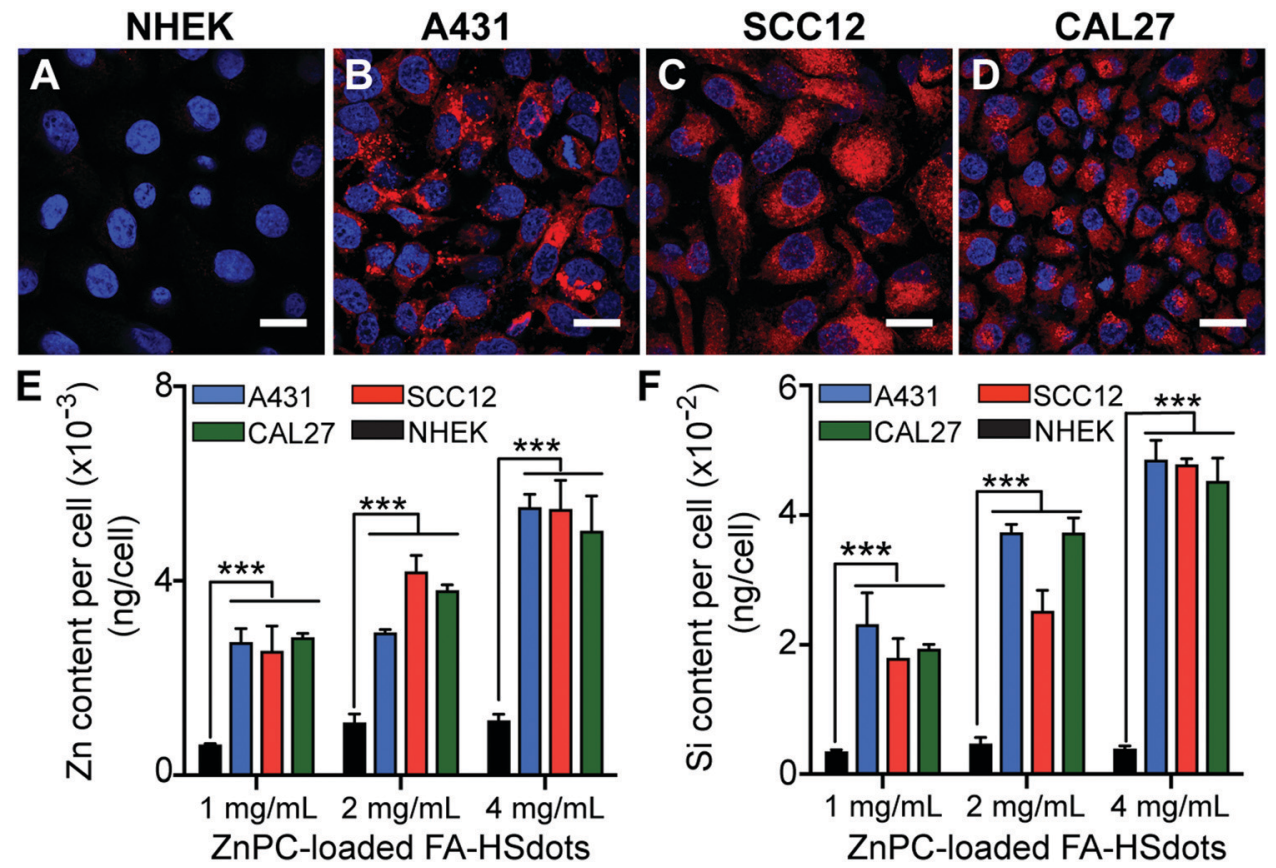

Fig. 3 Cellular uptake of ZnPC-loaded FA-HSdots. ZnPC-loaded FA-HSdots were labeled with TRITC. $1 \mathrm{mg} \mathrm{mL}^{-1}$ of ZnPC-loaded FA-HSdots was incubated with SCC cells or NHEKs for $48 \mathrm{~h}$. (A-D) Although TRITC was barely detectable in NHEKs (A), bright TRITC fluorescence was seen in the SCC cell lines (B-D). (E and F) Inductively coupled plasma-optical emission spectrometry (ICP-OES) showed 4-5-fold more Zn and 6-7-fold more Si in SCC cells compared to NHEKs. Scale bar: $15 \mu \mathrm{m} .{ }^{* *} p<0.001$.
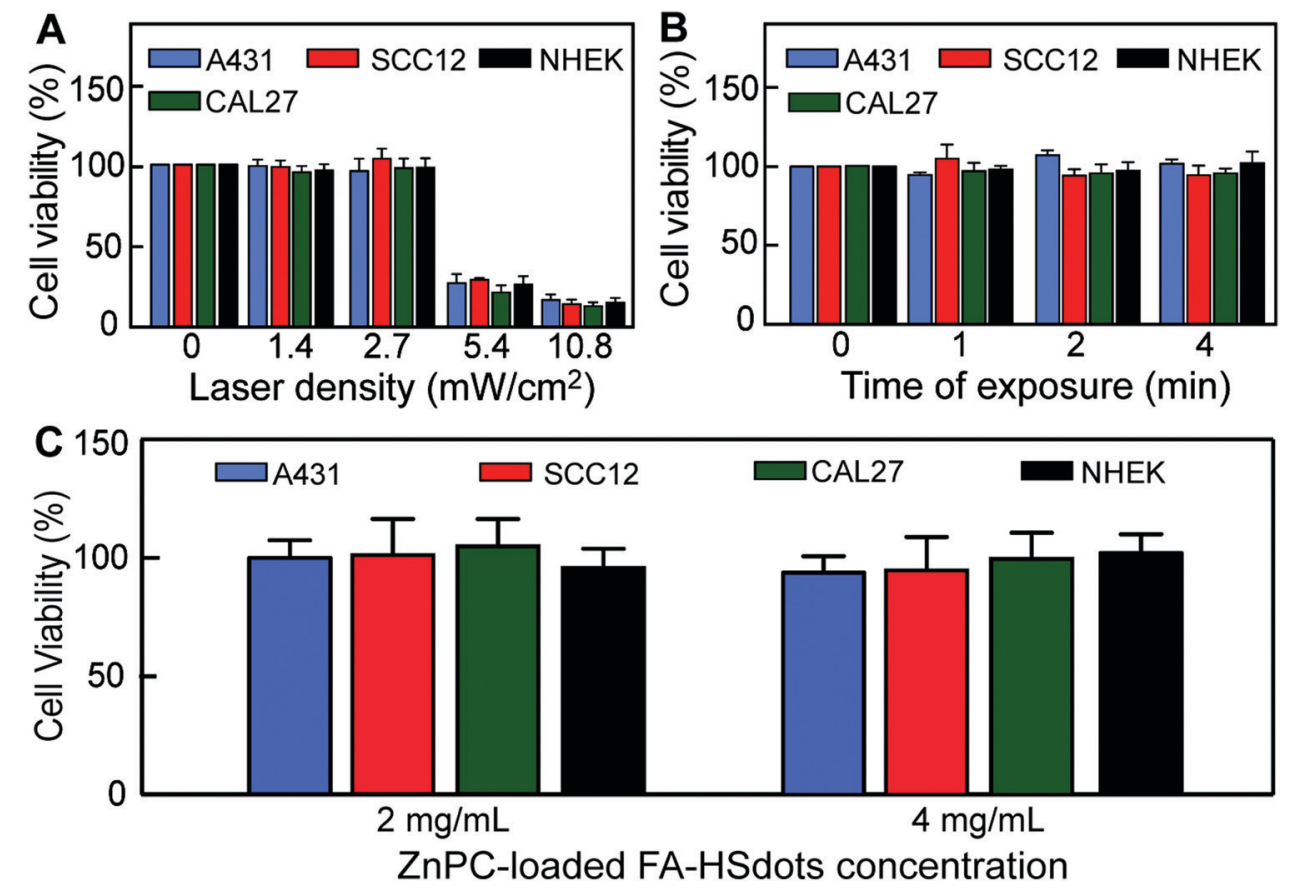

Fig. 4 Exposure to laser or ZnPC-loaded FA-HSdots alone does not cause cell toxicity. (A) Death was observed in $>70 \%$ of SCC cells and NHEKs after $1 \mathrm{~min}$ exposure to $5.4 \mathrm{~mW} \mathrm{~cm}{ }^{-2}$ or $10.8 \mathrm{~mW} \mathrm{~cm}^{-2}$ laser, but not after exposure to up to $2.7 \mathrm{~mW} \mathrm{~cm}^{-2}$ laser. (B) Even with 4 min of exposure at $2.7 \mathrm{~mW} \mathrm{~cm}^{-2}$, no cell death was observed. (C) 2 to $4 \mathrm{mg} \mathrm{mL}^{-1}$ of ZnPC-loaded FA-HSdots ( 0.02-0.04 $\left.\mathrm{mg} \mathrm{mL}^{-1} \mathrm{ZnPC}\right)$ without light irradiation for 48 hours caused no toxicity.

with a maximal cell death $(\geq 90 \%)$ achieved with $4 \mathrm{mg} \mathrm{mL}^{-1}$ of ZnPC-loaded FA-HSdots (Fig. 5A). NHEKs tolerated the treatment well $(<10 \%$ cell death) at all doses of ZnPC-loaded
FA-HSdots (Fig. 5A). At 24 hours after laser exposure, cell death occurred in more than $99 \%$ of SCCs treated with $4 \mathrm{mg} \mathrm{mL}^{-1}$ of ZnPC-loaded HSdots, but in fewer than $5 \%$ of similarly-treated 

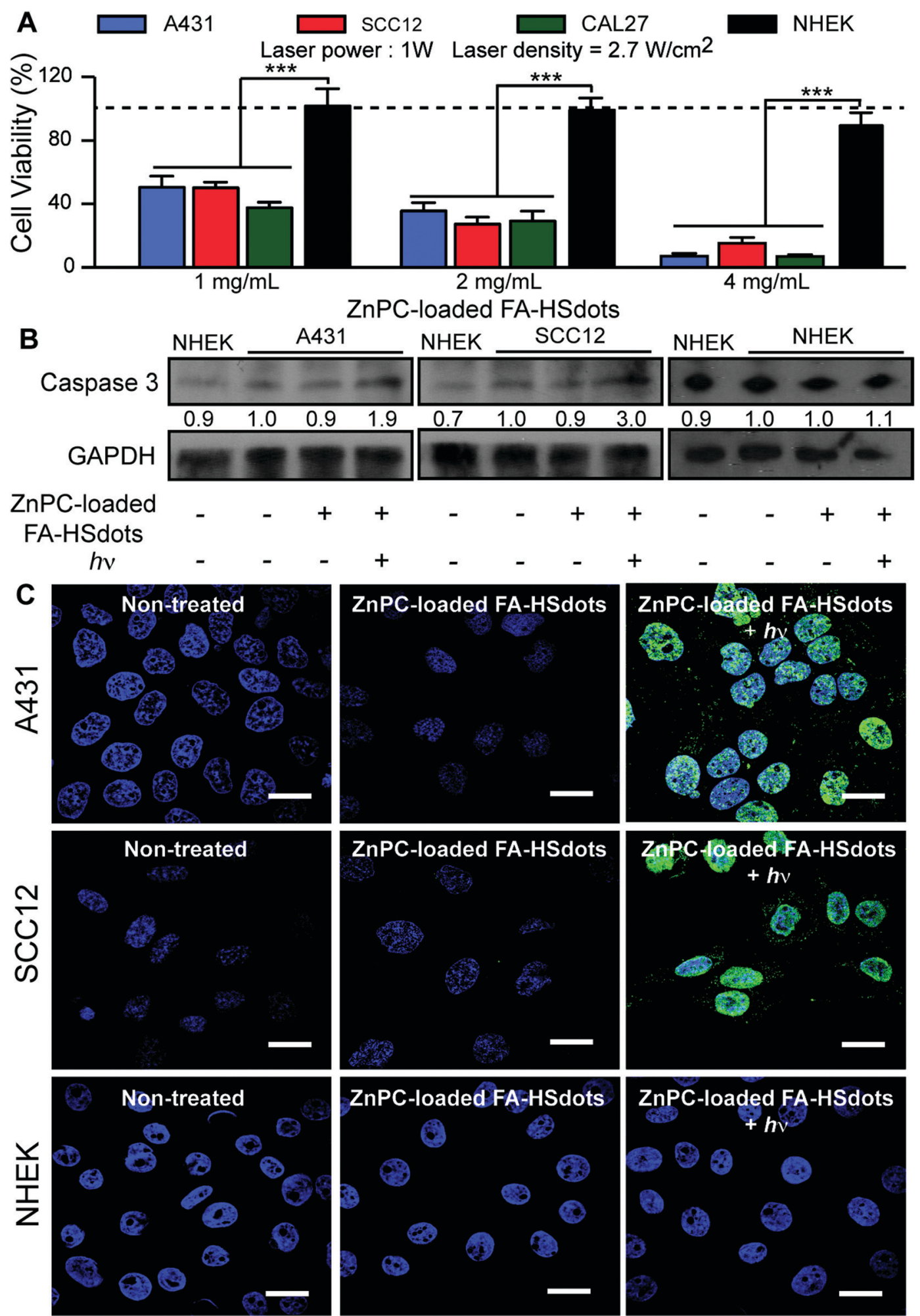

Fig. 5 Therapeutic efficacy of ZnPC-loaded FA-HSdots observed in all SCC cells after treatment with 2 min laser exposure ( $h \nu$ ). (A) At $4 \mathrm{mg} \mathrm{mL}^{-1}$ of ZnPC-loaded FA-HSdots, about $90 \%$ in vitro therapeutic efficacy ( $\geq 90 \%$ cell death) was achieved in SCC cells (A431 and SCC12 cells shown here), but not in NHEKs. (B) Increased caspase 3 expression in A431 and SCC12 cells treated with ZnPC-loaded FA-HSdots plus laser vs. SCC cells treated only with ZnPC-loaded FA-HSdots or NHEKs treated with both. (C) TUNEL assay shows an increase in nuclear DNA fragmentation, indicated by green signals, in SCC cells treated with ZnPC-loaded FA-HSdots and laser, but not in NHEKs. Scale bar: $15 \mu \mathrm{m}$. 
NHEKs (Fig. S6, ESI $\dagger$ ). Western blot assays showed a 2 to 3 -fold greater expression of caspase 3 after treatment of A431, SCC12 and CAL27 cells vs. NHEKs (Fig. 5B). Furthermore, terminal deoxynucleotidyl transferase (TdT) dUTP Nick-End Labeling (TUNEL) assays showed more double-stranded DNA breaks in A431, SCC12 and CAL27 cells after treatment with nanoparticles plus laser, but not in NHEKs (Fig. 5C and Fig. S7, ESI $\dagger$ ). These results suggested that cancer cells selectively underwent apoptosis after concurrent treatment with $4 \mathrm{mg} \mathrm{mL}^{-1}$ of ZnPC-loaded FA-HSdots and $671 \mathrm{~nm}$ laser.

We then compared the therapeutic effect of ZnPC-loaded FA-HSdots with its free counterpart ZnPC and the commercially available 5-ALA. We incubated SCCs for 48 hours with $0.04 \mathrm{mg} \mathrm{mL}^{-1}$ of $\mathrm{ZnPC}$, a concentration greater than or equal to the concentration of $\mathrm{ZnPC}$ in $4 \mathrm{mg} \mathrm{mL}^{-1} \mathrm{ZnPC}$-loaded FA-HSdots, or $0.1 \mathrm{mg} \mathrm{mL}^{-1}$ of 5-ALA, which has previously been show to kill most cells in vitro. ${ }^{31,32}$ Without laser exposure, we observed no toxic effect of free ZnPC in either SCCs or NHEKs (Fig. S8a, ESI $\dagger$ ). Even with exposure to the $670 \mathrm{~nm}$ laser for $2 \mathrm{~min}$, cell death was minimal for SCC cells $(<10 \%)$ and around $20 \%$ in NHEKs with $0.04 \mathrm{mg} \mathrm{mL}^{-1}$ of free ZnPC (Fig. S8b, ESI $\dagger$ ), confirming the low therapeutic efficiency of free ZnPC and the importance of the FA-HSdots as a nanocarrier to enhance uptake and efficacy of ZnPC. Interestingly, 5 -ALA treatment alone caused death of only $<10 \%$ SCC cells, but $>90 \%$ of NHEKs (Fig. S8a, ESI $\dagger$ ). Even with laser exposure and the 5-ALA, fewer than $10 \%$ of the SCCs died, but the laser increased the toxicity of the 5-ALA to $>95 \%$ dead NHEKs (Fig. S8b, ESI $\dagger$ ), suggesting that our ZnPC-loaded FA-HSdots are less toxic to normal skin cells than 5-ALA.

Nanoparticle constructs are novel cancer therapeutic agents that deliver high local concentrations of drugs to tumors ${ }^{33,34}$ because of their biocompatibility and high surface area, which can be easily modified for drug loading. ${ }^{35}$ With suitable size (10-100 nm) and flexible surface properties, nanoparticle constructs are ideal to use for both passive or active targeting systems with superior tumor specificity than current drug methods. ${ }^{36}$ Passive targeting takes advantage of enhanced permeability and retention (EPR) effect due to the leaky vasculature in cancer cells and tumors, but not in healthy cells and tissues. ${ }^{37}$ Rapid growth of cancer cells requires fast vascularization, leading to vasculature that is more permeable to nanoparticles $(10-60 \mathrm{~nm})$ than normal tissues. Furthermore, poor lymphatic drainage in the tumor bed results in an accumulation of nanoparticles within the tumor and cancer cells. ${ }^{37}$ Through EPR, drug-loaded nanoparticles can improve the stability and accumulation of the drug in tumor. ${ }^{38,39}$ The size of our nanodots $(\sim 40 \mathrm{~nm})$ is within the range that is suitable for EPR, hence we believe that our nanodots conjugates can also potentially take advantage of EPR effects to deliver photosensitizer to the tumors in vivo.

Nanoparticles that are used in the active targeting of cancer cells are typically conjugated with a targeting moiety permitting preferential accumulation of the drug within selected tissues, individual cancer cells, or intracellular organelles that are associated with specific molecules in cancer cells. ${ }^{37}$ Because of the ability to target specifically cancer cells, nanoparticles with active targeting properties can improve therapeutic efficacy of the drugs and also decrease toxicity by reducing uptake in healthy tissues. ${ }^{39-42}$ Our ZnPC-loaded FA-HSdots also possess this property.

The folate receptor (FR) is highly expressed on the surface of epithelial, ovarian, cervical, breast, lung, kidney, colorectal, and brain tumors, ${ }^{43-45}$ but not sarcomas, lymphomas, and pancreatic, testicular, bladder, prostate, or liver cancers. ${ }^{44}$ In normal tissue, FR expression only presents in the apical surface of polarized epithelia in lungs, kidneys, placenta and choroid plexus, and its expression in significantly lower than in cancer cells and tumors. ${ }^{44}$ Folic acid (FA) is a non-immunogenic, small (441 Da), stable, inexpensive, and easy to synthesize molecule that has the ability to bind to FR with or without being conjugated onto nanoparticles. ${ }^{46}$ After attaching to the FR, FA-conjugated nanoparticles endocytose and accumulate in endosomes and lysosomes. ${ }^{46}$ In our study, we have introduced FA in our photosensitizer-loaded nanodots to increase the selectivity of the nanodots. As the results, we observed 5-6 fold increases in uptake of ZnPC-loaded FA-HSdots in SCC cells compared to in NHEKs. This phenomenon subsequently resulted in a therapeutic effect, which is only specific to cancer cells and not normal cells. Furthermore, distinct therapeutic efficacy was only observed with FA-ZnPC-loaded HSdots under laser exposure, suggesting that our ZnPC was activated by the laser and released to induce preferential cancer cell death. In contrast, without FA, ZnPC-loaded HSdots could be internalized by both SCC cells and NHEKs and resulted in similar toxicity to both cancer and normal cells after laser exposure.

We have previously reported that, in serological study of liver and kidney function indexes, photosensitizer-loaded HSdots showed no toxicity in animals. ${ }^{29}$ For greater clinical relevance, we selected ZnPC as the photosensitizer because of its absorption peak at $671 \mathrm{~nm}$ in the near infrared region, which can then penetrate deeply through the skin (up to $3 \mathrm{~mm}$ ). ${ }^{47}$ Free form of ZnPC has low solubility in PBS, while ZnPC-loaded FA-HSdots can be disperse in PBS solution easily and is stable in cold storage for an extended time. Our portable laser allows easy application to the clinical setting. The high PDT efficacy (more than $90 \%$ cancer cell death and strong evidence of apoptosis with $4 \mathrm{mg} \mathrm{mL}^{-1}$ ZnPC-loaded FA-HSdots and 2 min exposure to $671 \mathrm{~nm}$ laser) is largely attributable to the selective uptake of ZnPC-loaded FA-HSdots in the SCCs, sparing normal epidermal cells.

\section{Conclusions}

In conclusion, we have demonstrated a promising nanoconstruct that can be used to advance treatment of cutaneous squamous cell carcinoma. Our new approach for photodynamic therapy will be more efficacious, less invasive, and less toxic than current PDT approaches. Our nanoconstruct is not only stable and has a long shelf-life, but can also be topically applied and has the ability to target selectively SCC cells. Before advancing towards preclinical and human trials, we would like to develop a method to produce in situ SCC skin, in which we can not only test the penetration of the nanoconstruct into the skin, but also validate its therapeutic property through both topical application and intravenous injection. 


\section{Experimental}

Materials

Pluronic surfactant F108 $\left(\mathrm{PEO}_{132} \mathrm{PPO}_{50} \mathrm{PEO}_{132}\right.$, in which PEO represents polyethyleneoxide and PPO represents polypropyleneoxide), tetraethoxysilane (TEOS), dimethyldimethoxysilane (DEDMS), aminopropyl triethoxysilane (APES), folic acid (FA), $N, N^{\prime}$-dicyclohexylcarbodiimide (DCC), 4-dimethylaminopyridine (DMAP), tetramethyl rhodamine isothiocyanate (TRITC), zinc phthalocyanine (ZnPC) and other chemicals and solvents were purchased from Sigma-Aldrich. All chemicals involved in this study were used as received unless otherwise specified.

\section{Characterizations}

Transmission electron microscopy (TEM) images were captured on a JEOL JEM-1400 transmission electron microscope with operation voltage of $100 \mathrm{kV}$. Dynamic light scattering (DLS) and zeta potential experiments were performed at $25{ }^{\circ} \mathrm{C}$ using a Malvern Zetasizer NanoZS.

\section{Synthesis of folic acid-modified F108}

$1.46 \mathrm{~g}$ of F108, $0.176 \mathrm{~g}$ of FA and $0.025 \mathrm{~g}$ DMAP were dissolved in $50 \mathrm{~mL}$ DMSO and stirred in an ice bath for $30 \mathrm{~min}$ before $0.165 \mathrm{~g}$ DCC was added, and the mixture was allowed to react in an ice bath for $24 \mathrm{~h}$. Excessive reactants and impurities were removed by dialysis in DMSO and water. The modification rate of FA was determined to be $85 \%$ by elemental analysis on a EuroEA CHNS-O analyzer (EuroVector).

\section{Synthesis of hollow polymer-silica nanohybrid (HSdots)}

HSdots was synthesized as previously reported. $0.25 \mathrm{~g}$ F108, $0.112 \mathrm{~g}$ of $\mathrm{KCl}$ were solubilized in $7.5 \mathrm{~mL}$ of $2 \mathrm{M} \mathrm{HCl}$ and stirred for $1 \mathrm{~h}$. Then $240 \mu \mathrm{L}$ of cyclohexane was added and stirred for another $1 \mathrm{~h}$, before the addition of $268 \mu \mathrm{L}$ TEOS and then, $4 \mathrm{~h}$ later, $80 \mu \mathrm{L}$ DEDMS. After being stirred for another $24 \mathrm{~h}$, the product was dialyzed in deionized water 4 times (25000 Da molecular weight membrane). Cyclohexane was removed at $50{ }^{\circ} \mathrm{C}$ under vacuum and the solution was filtered by a $0.2 \mu \mathrm{M}$ membrane filter before further application or characterization.

Synthesis of hollow polymer-silica nanohybrid with FA ligands (FA-HSdots)

In the synthesis of FA-HSdots, ${ }^{29}$ FA-F108 was doped in F108 in a weight ratio of $5 \%$ as surfactant $(0.0125 \mathrm{~g}$ FA-F108 was mixed with $0.2375 \mathrm{~g}$ F108), and solubilized in $7.5 \mathrm{~mL}$ of $2 \mathrm{M} \mathrm{HCl}$ together with $0.112 \mathrm{~g}$ of $\mathrm{KCl}$.

\section{Fluorescent labeling with TRITC}

To label the surface of HSdots or FA-HSdots, $1 \mathrm{mg}$ of TRITC was dissolved in $0.2 \mathrm{~mL}$ anhydrous dimethyl formamide (DMF) with $1.6 \mu \mathrm{L}$ APES and stirred at room temperature for $24 \mathrm{~h}$. The mixture was then added to $10 \mathrm{~mL}$ of $20 \mathrm{mg} \mathrm{mL}^{-1}$ HSdots or FA-HSdots solution with $0.1 \mathrm{~mL}$ ammonia. After $24 \mathrm{~h}$, the product was purified by dialysis in $1000 \mathrm{~mL}$ deionized water 8 times until the water became colorless.

\section{Loading of ZnPC}

ZnPC (1.0 mg) was solubilized in dichloromethane (DCM, $50 \mu \mathrm{L}$ ) and then added to a $10 \mathrm{~mL}$ aqueous dispersion containing $10 \mathrm{mg} \mathrm{mL}{ }^{-1}$ HSdots or FA-HSdots. The mixture was sonicated to form a homogenous solution. DCM was removed by rotary evaporation at $50{ }^{\circ} \mathrm{C}$ under 150 mbar.

\section{Statistical analysis}

Student's $t$-test was used for all analyses. $p<0.05$ was considered significant. All studies were performed at least 3 times in triplicate.

\section{Cell culture and human samples}

NHEKs were isolated from neonatal foreskin, as previously described (Li et al., 1998), and cultured in keratinocyte serumfree medium (Cascade Biologics, Portland, OR) supplemented with $0.07 \mathrm{mM} \mathrm{CaCl}_{2}$ unless otherwise indicated. Cancer cells were grown in DMEM/F12 (Cascade Biologics) with 10\% FBS (Cascade Biologics). All cultures were maintained at $37{ }^{\circ} \mathrm{C}$ in $5 \%$ $\mathrm{CO}_{2}$. Human skin and human SCC tissues were obtained from the Northwestern SDRC Repository after IRB-approved written consent according to the Declaration of Helsinki guidelines.

\section{Confocal imaging}

Cells were cultured on glass coverslips in 12-well plates to $50-60 \%$ confluence. After pre-treatment with ZnPC-loaded FA-HSdots, cells were washed with PBS, fixed for $15 \mathrm{~min}$ in $4 \%$ paraformaldehyde, stained with $1 \mu \mathrm{M}$ DAPI for $10 \mathrm{~min}$ at room temperature (RT), and mounted onto slides in mounting medium (Prolong Gold antifade reagent, Life Technologies, Grand Island, NY).

\section{Histology}

Serial sections of paraffin-embedded tissues were cut for histology and immunofluorescence analysis. Hematoxylin and eosin stained $4 \mu \mathrm{m}$ sections were photographed at $10 \times$ magnification under light microscopy (Zeiss Axioplan 2 imaging, Thornwood, NY), and imaged digitally (Axiovision version 4.5).

\section{Immunofluorescence}

Deparaffinization of paraffin-embedded human tissues sections was carried out in xylene, followed by an ethanol gradient (100-70\%). Antigen retrieval was conducted in citrate buffer (pH 6.0) at $99{ }^{\circ} \mathrm{C}$ for $60 \mathrm{~min}$. All samples were then blocked with $5 \%$ goat serum, $2 \%$ human serum and $1 \%$ BSA in PBS, and incubating with anti-folate receptor antibody overnight at $4{ }^{\circ} \mathrm{C}$. After treatment with fluorescence-conjugated secondary antibodies for $1 \mathrm{~h}$ at room temperature and mounting with DAPI-containing medium, sections were viewed by Nikon A1R confocal fluorescence microscopy (NU Center for Advanced Microscopy/Nikon Imaging Center).

\section{Western blot}

Total cell lysates were extracted in boiling buffer containing $10 \mathrm{mM}$ Tris ( $\mathrm{pH} 7.4$ ), $1 \% \mathrm{SDS}$, and $1 \mathrm{mM} \mathrm{Na}_{3} \mathrm{VO}_{4}$ (Sigma). The solution was centrifuged at $12000 \mathrm{rpm}$ for $10 \mathrm{~min}$ at RT to 
remove debris, and protein concentrations were determined using the BCA Protein Assay Kit (Thermo Fisher Scientific). Equal amounts of proteins were subjected to 10\% SDS-PAGE, transferred to a nitrocellulose membrane, and blocked for $1 \mathrm{~h}$ in Tris-buffered saline, $\mathrm{pH}$ 7.5, containing 0.5\% Tween 20 and $5 \%$ milk. Membranes were then incubated overnight at $4{ }^{\circ} \mathrm{C}$ with anti-folate receptor antibody or anti-caspase 3 antibody (Santa Cruz Biotechnology, Santa Cruz, CA) for 24 hours. The membranes were then incubated with horseradish peroxidaseconjugated secondary antibody (Jackson ImmunoResearch Laboratories, West Grove, PA) for $1 \mathrm{~h}$, and the bound antibodies were detected using chemiluminescence (Pierce Chemical Co., Rockford, IL). Images were digitized and band densities were measured using NIH-Image software.

\section{Inductively coupled plasma optical emission spectrometry (ICP-OES)}

$200 \mu \mathrm{L} \mathrm{HNO}_{3}$ was added to each sample, which was then digested at room temp for $1.5 \mathrm{~h}$ and at $65{ }^{\circ} \mathrm{C}$ for $45 \mathrm{~min}$. After subsequent digestion at room temperature overnight, $250 \mu \mathrm{L}$ of each digest was transferred to a $15 \mathrm{~mL}$ metal-free tube (Falcon, Corning, NY) and diluted to $10 \mathrm{~mL}$ with $0.05 \% \mathrm{HF}$ in $\mathrm{H}_{2} \mathrm{O}$. Zinc and silicon contents were measured using ICP-OES. Zinc and silicon contents of each sample were then normalized to the total number of cells in the sample.

\section{TUNEL assay}

Cells were cultured on glass coverslips in 12-well plates to 50-60\% confluence. After pre-treatment with ZnPC-loaded FA-HSdots, cells were washed with PBS and fixed for $15 \mathrm{~min}$ in $4 \%$ paraformaldehyde. The DeadEnd Fluorometric TUNEL system assay was used to measure the nuclear fragmented DNA of apoptotic cells. The assay catalytically incorporates fluorescein-12-dUTP at the $3^{\prime}$-OH DNA ends using recombinant terminal deoxynucleotidyl transferase (rTdT), which forms a polymeric tail in the TdT-mediated dUTP Nick-End labeling (TUNEL) assay. Once TUNEL assay was performed, the nuclei of the cells were counterstained with DAPI. The cells were then visualized by confocal microscopy.

\section{Conflicts of interest}

There are no conflicts to declare.

\section{Acknowledgements}

This work was supported by NTU-Northwestern Institute for Nanomedicine (AP, YZ), the National Institutes of Health Grants R01AR068375 (AP), the Postgraduate Training in Cutaneous Biology T32 AR060710 (DD), and the Singapore National Research Foundation Investigatorship NRF-NRFI2018-03 (YZ). This research also utilized Core resources provided by the Northwestern University Skin Disease Research Center (NIAMS, P30AR057216) and the Northwestern University Center for Advanced Microscopy (NCI, P30CA060553). Metal analysis was performed at the
Northwestern University Quantitative Bio-element Imaging Center and was funded by the Chicago Biomedical Consortium with support from the Searle Funds at The Chicago Community Trust and supported by the National Institutes of Health under Award Number S10OD020118.

\section{Notes and references}

1 A. G. Glass and R. N. Hoover, J. Am. Med. Assoc., 1989, 262, 2097-2100.

2 V. Madan, J. T. Lear and R. M. Szeimies, Lancet, 2010, 375, 673-685.

3 E. Pukkala, P. Noksokoivisto and P. Roponen, Int. Arch. Occup. Environ. Health, 1992, 64, 39-42.

4 P. Agostinis, K. Berg, K. A. Cengel, T. H. Foster, A. W. Girotti, S. O. Gollnick, S. M. Hahn, M. R. Hamblin, A. Juzeniene, D. Kessel, M. Korbelik, J. Moan, P. Mroz, D. Nowis, J. Piette, B. C. Wilson and J. Golab, Ca-Cancer J. Clin., 2011, 61, 250-281. 5 E. Buytaert, M. Dewaele and P. Agostinis, Biochim. Biophys. Acta, Rev. Cancer, 2007, 1776, 86-107.

6 J. P. Taquet, C. Frochot, V. Manneville and M. BarberiHeyob, Curr. Med. Chem., 2007, 14, 1673-1687.

7 S. J. Yang, C. F. Lin, M. L. Kuo and C. T. Tan, Biomacromolecules, 2013, 14, 3183-3191.

8 J. de Leeuw, N. van der Beek, W. D. Neugebauer, P. Bjerring and H. A. Neumann, Lasers Surg. Med., 2009, 41, 96-103.

9 Q. Peng, T. Warloe, K. Berg, J. Moan, M. Kongshaug, K. E. Giercksky and J. M. Nesland, Cancer, 1997, 79, 2282-2308.

10 C. S. Souza, L. B. Felicio, J. Ferreira, C. Kurachi, M. V. Bentley, A. C. Tedesco and V. S. Bagnato, Photodiagn. Photodyn. Ther., 2009, 6, 207-213.

11 S. R. Wiegell, H. C. Wulf, R. M. Szeimies, N. Basset-Seguin, R. Bissonnette, M. J. Gerritsen, Y. Gilaberte, P. CalzavaraPinton, C. A. Morton, A. Sidoroff and L. R. Braathen, J. Eur. Acad. Dermatol. Venereol., 2012, 26, 673-679.

12 K. Szocs, G. Csik, A. D. Kaposi and J. Fidy, Biochim. Biophys. Acta, 2001, 1541, 170-178.

13 P. K. Lee and A. Kloser, J. Drugs Dermatol., 2013, 12, 925-930.

14 C. Ash, M. Dubec, K. Donne and T. Bashford, Lasers Med. Sci., 2017, 32, 1909-1918.

15 C. Wong, T. Stylianopoulos, J. A. Cui, J. Martin, V. P. Chauhan, W. Jiang, Z. Popovic, R. K. Jain, M. G. Bawendi and D. Fukumura, Proc. Natl. Acad. Sci. U. S. A., 2011, 108, 2426-2431.

16 I. Brigger, C. Dubernet and P. Couvreur, Adv. Drug Delivery Rev., 2002, 54, 631-651.

17 H. Chen, Z. Zhen, T. Todd, P. K. Chu and J. Xie, Mater. Sci. Eng., $R$, 2013, 74, 35-69.

18 D. H. Dam, R. C. Lee and T. W. Odom, Nano Lett., 2014, 14, 2843-2848.

19 M. E. Davis, Z. G. Chen and D. M. Shin, Nat. Rev. Drug Discovery, 2008, 7, 771-782.

20 Y. Liu, H. Miyoshi and M. Nakamura, Int. J. Cancer, 2007, 120, 2527-2537.

21 M. Longmire, P. L. Choyke and H. Kobayashi, Nanomedicine, 2008, 3, 703-717. 
22 Y. Zhang, C. Y. Ang, M. Li, S. Y. Tan, Q. Qu, Z. Luo and Y. Zhao, ACS Appl. Mater. Interfaces, 2015, 7, 18179-18187.

23 Y. Zhang, C. Y. Ang, M. Li, S. Y. Tan, Q. Qu and Y. Zhao, ACS Appl. Mater. Interfaces, 2016, 8, 6869-6879.

24 Y. Zhang, Q. Qu, X. Cao and Y. Zhao, Chem. Commun., 2017, 53, 12032-12035.

25 G. L. Zwicke, G. A. Mansoori and C. J. Jeffery, Nano Rev., 2012, 3, 18496.

26 D. Shen, J. Yang, X. Li, L. Zhou, R. Zhang, W. Li, L. Chen, R. Wang, F. Zhang and D. Zhao, Nano Lett., 2014, 14, 923-932.

27 A. Kunzmann, B. Andersson, T. Thurnherr, H. Krug, A. Scheynius and B. Fadeel, Biochim. Biophys. Acta, 2011, 1810, 361-373.

28 L. Z. Zhao, W. Yuan, C. Y. Ang, Q. Y. Qu, Y. Dai, Y. L. Gao, Z. Luo, J. G. Wang, H. Z. Chen, M. H. Li, F. Y. Li and Y. L. Zhao, Adv. Funct. Mater., 2016, 26, 3036-3047.

29 L. Zhao, W. Yuan, H. P. Tham, H. Chen, P. Xing, H. Xiang, X. Yao, X. Qiu, Y. Dai, L. Zhu, F. Li and Y. L. Zhao, Small, 2017, 13, 1700963.

30 M. Sakamoto, Laser Ther., 2012, 21, 60-64.

31 P. Xing and Y. L. Zhao, Adv. Mater., 2016, 28, 7304-7339.

32 M. El-Khatib, C. Tepe, B. Senger, M. Dibue-Adjei, M. J. Riemenschneider, W. Stummer, H. J. Steiger and J. F. Cornelius, Int. J. Mol. Sci., 2015, 16, 9936-9948.

33 L. A. Dykman and N. G. Khlebtsov, Chem. Rev., 2014, 114, 1258-1288.
34 A. Z. Wang, R. Langer and O. C. Farokhzad, Annu. Rev. Med., 2012, 63, 185-198.

35 J. Xie, S. Lee and X. Chen, Adv. Drug Delivery Rev., 2010, 62, 1064-1079.

36 S. A. Mousa and D. J. Bharali, Cancers, 2011, 3, 2888-2903.

37 R. Sinha, G. J. Kim, S. Nie and D. M. Shin, Mol. Cancer Ther., 2006, 5, 1909-1917.

38 A. G. Cuenca, H. Jiang, S. N. Hochwald, M. Delano, W. G. Cance and S. R. Grobmyer, Cancer, 2006, 107, 459-466.

39 G. F. Paciotti, L. Myer, D. Weinreich, D. Goia, N. Pavel, R. E. McLaughlin and L. Tamarkin, Drug Delivery, 2004, 11, 169-183.

40 D. H. Dam, J. H. Lee, P. N. Sisco, D. T. Co, M. Zhang, M. R. Wasielewski and T. W. Odom, ACS Nano, 2012, 6, 3318-3326.

41 M. A. Dobrovolskaia and S. E. McNeil, Nat. Nanotechnol., 2007, 2, 469-478.

42 M. V. Yezhelyev, X. Gao, Y. Xing, A. Al-Hajj, S. Nie and R. M. O'Regan, Lancet Oncol., 2006, 7, 657-667.

43 P. Garinchesa, I. Campbell, P. E. Saigo, J. L. Lewis, L. J. Old and W. J. Rettig, Am. J. Pathol., 1993, 142, 557-567.

44 N. Parker, M. J. Turk, E. Westrick, J. D. Lewis, P. S. Low and C. P. Leamon, Anal. Biochem., 2005, 338, 284-293.

45 H. S. Yoo and T. G. Park, J. Controlled Release, 2004, 100, 247-256.

46 C. Muller and R. Schibli, J. Nucl. Med., 2011, 52, 1-4.

47 T. A. Henderson and L. D. Morries, Neuropsychiatr. Dis. Treat., 2015, 11, 2191-2208. 